\title{
Testing the cosmic ray content in galaxy clusters
}

\author{
P. Marchegiani ${ }^{1}$, G. C. Perola ${ }^{1}$, and S. Colafrancesco ${ }^{2}$ \\ 1 Dipartimento di Fisica, Università Roma 3, via della Vasca Navale 84, Roma, Italy \\ e-mail: [marchegiani; perola]@fis.uniroma3.it \\ 2 INAF - Osservatorio Astronomico di Roma via Frascati 33, 00040 Monteporzio, Italy
}

Received 5 July 2006 / Accepted 18 December 2006

\begin{abstract}
Context. The effective content of cosmic rays $(C R)$ in galaxy clusters remains elusive. The evidence of relativistic electrons $(R E)$ in the subset of clusters endowed with a radio halo remains hardly quantitative in the absence of robust estimates of the magnetic field $B(r)$, derived from Faraday Rotation (FR) measurements. The content in relativistic protons $(R P)$ requires a different approach, the only direct one residing in the detection of their collisional production of gamma rays $(G R)$.

Aims. Based on the evidence of merging phenomena in clusters, theory predicts a large content of $R P$, whose energy density could be a large fraction of the thermal energy. This paper aims to estimate a maximum production of both secondary relativistic electrons, $S R E$, and $G R$ from the $R P$ that have supposedly accumulated throughout the entire history of a cluster.

Methods. SRE and GR production is maximized when the $R P$ and the thermal gas share the same radial profile. The production rate is normalized by adopting a reference value of 0.3 for $\xi$, the ratio of $R P$ to thermal pressure. The $S R E$ content which obtains, when constrained to reproduce the observed radio brightness profile, yields univocally $B(r)$, if the presence of primary $R E$ were negligible. Results. This procedure is applied to four radio-halo clusters (Coma, A2163, A2255, A2319). In these objects, the central value $B_{0}$ required is consistent with typical, albeit rather uncertain, values derived from FR, although for A2163 and A2319 no reliable FR estimates are available to strengthen this result. On the other hand, $B(r)$ typically increases beyond the thermal core, a hardly acceptable condition. This problem is alleviated by assuming a mix of $S R E$ and of "primary" $R E$ ( $P R E$ ), with the latter becoming the dominant component beyond the thermal core. These results suggest that in clusters without a radio halo detected so far a diffuse radio-emission should also be observable due to SRE alone, and therefore more centrally condensed, provided that $\xi$ is of the order of 0.3. To encourage deeper radio observations of such clusters, some examples were selected that seem rather promising. Efforts in this direction, if accompanied by FR measurements, could provide highly significant constraints on the $C R$ content in clusters, even before the future GLAST mission will have accomplished the hard task of detecting the $G R$. A complementary result concerns the excess far UV in the Coma cluster, that some authors have attributed to IC emission from SRE. It is shown that this hypothesis can be excluded, because it requires a $R P$ energy content in excess of the thermal one.
\end{abstract}

Key words. galaxies: clusters: general

\section{Introduction}

A significant fraction of galaxy clusters are endowed with a diffuse, low-brightness radio-emission, which is clearly distinct from the extended radio components, if present, associated with member galaxies. The historical prototype (Willson 1970) is the radio source Coma $\mathrm{C}$ in the Coma cluster. These so-called radio halos represent direct evidence of relativistic electrons and magnetic fields embedded in the thermal plasma responsible for the X-ray emission. It is very likely that relativistic protons are present as well. In the following these components will be labelled $R E$ (electrons), $R P$ (protons), $C R$ (for cosmic rays, protons and electrons), and $B$ (the magnetic field and its strength). Since the brightness of the radio emission depends (non-linearly) on $B$ and the $R E$ density, it cannot be excluded that the presence of a non-thermal component is common to all clusters, including those without, as yet, an observed radio-halo.

Generally speaking, we are still far from solid quantitative estimates of the non-thermal content. The radio emission alone can only provide a minimum for the energy content in $C R$ and $B$ combined, according to the recipe first proposed by Burbidge (1956). This minimum is well below the thermal energy content (Govoni \& Feretti 2004).
A direct way to estimate the $R E$ and $B$ contents separately was first applied by Perola \& Reinhardt (1972) to Coma C. Although at the time they could (incorrectly, as it became clear later) and did attribute the whole of the X-ray emission to this process, the $R E$ inevitably lose energy on the photons of the cosmic microwave background through the socalled inverse Compton (IC) process, thus it remains obviously true that, within a rather wide range of $B$ values, the energy lost should be observed in the X-ray band. All the attempts, made so far, to disentangle this contribution from the thermal X-ray emission, which have concentrated attention on the hard (greater than $10-30 \mathrm{keV}$ ) regime, have led to results that are rather controversial particularly in their interpretation (Fusco-Femiano et al. 2004; Rossetti \& Molendi 2004). This follows from the evidence of an excess, possibly IC emission, obtained with non-imaging instruments, which therefore can only be used to estimate the volumeintegrated amount of $R E$ within the instrument's field of view and, rigorously speaking, only in a very narrow energy interval, 1.6-3.2 GeV. In a previous paper (where most references can be found, Colafrancesco et al. 2005, Paper I), we discussed this issue at length and emphasized the need to measure the surface brightness distribution of the IC emission, in order, first, to verify whether the $R E$ responsible for this 
radiation are effectively confined within the observed radio halo and, second, to solve the degeneracy intrinsic to the synchrotron emission and therefore obtain fundamental information on the radial dependence of the $R E$ density and of $B$.

The origin of the $R E$ was outside the goal of Paper I. They can have a "primary" origin, which is accelerated along with the $R P$. However, there must also be $R E$ of "secondary" origin, produced through the inevitable inelastic interactions of the $R P$ with the ambient plasma. This process, notably, gives rise to the emission of gamma rays $(G R)$ with energy $\geq 50 \mathrm{MeV}$. If we knew the number and distribution of the $R E$ (from their IC emission), the question (Dennison 1980) whether the "secondary" ones (SRE) are either a negligible or an important fraction of the whole $R E$ population would require assumptions on the amount of $R P$, with basically one single important constraint, namely that their pressure should not exceed that of the thermal gas. Vice versa, if by measuring the spectrum, flux, and the associated surface brightness of the gamma rays (after verifying that they cannot be due to other mechanisms, such as the hypothetical annihilation process of the dark matter, see for instance Pieri \& Branchini 2004; Colafrancesco et al. 2006) one could derive the amount of $R P$, a very stringent constraint on the production rate of the $S R E$ could be placed. If this rate were too low for a significant contribution to the radio-emission, except for unacceptably high values of $B$, the answer to the question would be immediate. It is clear, however, that only the combination of reliable measurements of the $G R$ and the IC X-rays, with the latter leading to an absolute estimate of the $R E$ amount and distribution, will close the circle. The amount and distribution of the $R E$ could also be obtained via an independent estimate of $B$ (radial distribution included), which can be derived from Faraday rotation (FR) measurements, once this goal is reached in an uncontroversial way (see Govoni \& Feretti 2004, for a discussion of the state of this art). In either (IC or FR) case, the $G R$ represent an irreplaceable step for the issue of the $C R$ content.

In this context, the contribution that the present paper aims to provide, given the still immature observational status, consists in evaluating the "maximal" requirements on the $R P$ and on $B$, including their radial distribution, assuming that the radioemission is "totally" due to the $S R E$. These requirements, constrained to avoid conflicts with either physical arguments (the non-thermal pressure should not exceed the thermal pressure) or observational evidence (limits on $B$ from FR), will be used to predict the uppermost value of the $G R$ emission intensity, which one would expect to inevitably follow.

The authors are aware of the fact that arguments have been put forward that are against a dominant contribution to the $R E$ of the SRE component (e.g. Brunetti 2003). Nonetheless, the approach adopted is useful on two different grounds. First, the $R E$ lifetime in the energy range of interest is short (about one tenth) compared to the age of a cluster; thus the currently relevant primary $R E(P R E)$ must have been accelerated in this last fraction of the cluster age, while the $R P$ giving rise to the $S R E$ in the same fraction of time have likely accumulated over the entire cluster lifetime, meaning that their contribution to the $R E$ content might be far from negligible with respect to that due to the acceleration processes. Second, the choice of maximizing this contribution leads in a sense to the most optimistic predictions on the intensity of the $G R$ emission, which could be immediately tested by its measurement.

Another item is connected with the excess emission reported in the far ultraviolet-soft X-rays in the Coma cluster in particular, that might be attributed to IC emission. If this were the case, the relevant $R E$ population would fall into a totally different range of energies with respect to the radio-emitting ones. In this hypotheses, some authors (Bowyer et al. 2004) have attributed a "secondary" origin to these $R E$. Its relevance to the ensuing requirements on the parent $R P$ will also be discussed.

The paper is organized as follows. Section 2 contains a brief overview of the theoretical results on the acceleration of $R P$ in clusters. Section 3 is devoted to the assumptions and prescriptions on which the subsequent calculations are based. Section 4 illustrates the properties of the four clusters used as examples and the way their radio brightness distribution is described analytically. In Sect. 5 the results are reported for the $G R$ emission and for the strength and radial distribution of $B$, when the radio halo is "totally" attributed to the SRE. Section 6 is devoted to the issue of the UV excess in the Coma cluster as due to IC from $S R E$. Section 7 is devoted to a discussion, and Sect. 8 to the conclusions.

\section{Acceleration and accumulation of $R P$}

Since Jaffe (1977) discussed the possible diffuse origin (that is, in situ acceleration versus injection by radio sources) of the $R E$ in Coma $\mathrm{C}$, science has progressed a long way. In the theoretical scenarios of structure formation in the Universe through hierarchical clustering, with the relatively recent support from Chandra and XMM-Newton observations, the merging processes are widely suspected to be the events that can lead to a diffuse acceleration of $C R$ in clusters, along with an increase in the energy content of the thermal gas.

The papers quoted below do refer to the results (Blandford $\&$ Eichler 1987) of the shock diffusive acceleration theory that predict spectral indices $s$ linked to the Mach number $M$ as

$s=2 \frac{M^{2}+1}{M^{2}-1}$

From hydrodynamical simulations (e.g. Ryu et al. 2003) it appears that in the central regions, where the largest amount of particles are processed, the typical values of the Mach number are $2 \leq M \leq 4$, and, according to Eq. (1), those of spectral index are $2.3 \leq s \leq 3.3$. The amount of energy going into the $C R$ channel can be as large as 30-50\% of what goes into the thermal channel (Miniati et al. 2001a; Ryu et al. 2003). Much higher values of $M$, which are even more efficient in the acceleration process, are attained in the cluster outskirts, where (Pfrommer et al. 2006) a higher ratio between the two energy contents could eventually prevail, except that dynamical stability problems might then ensue. In this paper, as explained in the next section, care is taken that nowhere within the radio halos, as defined by the outermost contour of the available maps, does the non-thermal pressure (inclusive of $C R$ and $B$ ) exceed the thermal pressure. Furthermore, a single value of $s$ is adopted for simplicity, namely $s=2.7$. (Incidentally, this is the value required for reproducing with a $S R E$ population the radio slope of Coma C, the only one well determined among the four cases which will be considered in the following). A value of $s$ higher than 2 implies that the choice of the minimum energy is critical to the $C R$ pressure. Following Gieseler et al. (2000), the minimum adopted Lorentz factor is given by

$\gamma_{\min } \approx 1+\left[3.4 \times 10^{-5} k T_{\mathrm{keV}}\left(\frac{c_{2}}{4}\right)^{2}\right]$,

where $k T_{\mathrm{keV}}$ is the thermal gas temperature, and $c_{2}$ is approximately equal to 4 . Thus $\gamma_{\min }$ is practically equal to 1 in all cases. 
For the $R P$ momentum ( $p$ ) spectrum, the form adopted is $N_{\mathrm{RP}}(p) \propto p^{-s}$, which can be written as a function of $\gamma$ :

$N_{\mathrm{RP}}(\gamma, r)=K_{\mathrm{RP}}(r) \frac{\gamma}{\left(\gamma^{2}-1\right)^{(s+1) / 2}}$.

The associated pressure is given by

$P_{\mathrm{RP}}(r)=\frac{1}{3} m_{\mathrm{p}} c \int_{\gamma_{\min }}^{\gamma_{\max }} N_{\mathrm{RP}}(\gamma, r) \beta \gamma v(\gamma) \mathrm{d} \gamma$

The radial dependence of the RP content, with a constant $s$, as anticipated, will be described through that of their $P_{\mathrm{RP}}$.

\section{Assumptions and prescriptions}

The production rates per unit volume of the $S R E, Q_{\mathrm{e}}\left(\gamma_{\mathrm{e}}, r\right)$ and of the $G R, Q_{\mathrm{g}}\left(E_{\mathrm{g}}, r\right)$, which are strictly related to each other, are proportional to the product of the thermal and non-thermal proton density. Since the first of the two quantities is known, the radial behavior of the second, which maximizes the production rates, must be found.

According to what has been described in Sect. 2, and assuming that among the $C R$ the $R P$ are the energetically dominant component, it is convenient to introduce the ratio between $P_{\mathrm{RP}}$ and $P_{\mathrm{th}}$, the pressures of the non thermal and the thermal components,

$\xi(r)=\frac{P_{\mathrm{RP}}(r)}{P_{\mathrm{th}}(r)}$,

which is bound to remain everywhere less than unity:

$\xi(r) \leq 1$.

So long as $k T$ can be regarded as constant (a reasonably good description of clusters within the extent of their radio halos), and under the assumption that the mean $R P$ energy is also constant (a direct consequence of the assumption of a constant value of the exponent $s$ anticipated in Sect. 2), $\xi(r) \propto n_{\mathrm{RP}} / n_{\mathrm{th}}$, where $n_{\mathrm{RP}}\left(\equiv \int N_{\mathrm{RP}} \mathrm{d} \gamma\right)$ and $n_{\mathrm{th}}$ are the number densities of the relativistic and the thermal protons, respectively. Without loss of generality, the radial dependence of $n_{\mathrm{RP}}$ can be chosen with the same functional form as is generally used for $n_{\mathrm{th}}$,

$n_{\mathrm{th}}(r)=n_{\mathrm{th}, 0}\left[1+\left(\frac{r}{r_{\mathrm{c}, \mathrm{th}}}\right)^{2}\right]^{-q_{\mathrm{th}}}$,

with the two parameters, core radius and exponent, left free:

$n_{\mathrm{RP}}(r)=n_{\mathrm{RP}, 0}\left[1+\left(\frac{r}{r_{\mathrm{c}, \mathrm{RP}}}\right)^{2}\right]^{-q_{\mathrm{RP}}}$.

Not surprisingly, the production rates reach their maximum values when $r_{\mathrm{c}, \mathrm{RP}}=r_{\mathrm{c}, \text { th }}$ and $q_{\mathrm{RP}}=q_{\mathrm{th}}$, therefore for $\xi$ constant with radius, which will be labelled $\xi_{0}$. These values are given by

$$
\begin{aligned}
Q_{\mathrm{e}}\left(\gamma_{\mathrm{e}}, r\right)= & n_{\mathrm{th}, 0} K_{\mathrm{RP}, 0}\left[1+\left(\frac{r}{r_{\mathrm{c}, \mathrm{th}}}\right)^{2}\right]^{-2 q_{\mathrm{th}}} \int_{\gamma_{\mathrm{min}}}^{\infty} c \beta\left(\gamma_{\mathrm{p}}\right) \\
& \times \frac{\gamma_{\mathrm{p}}}{\left(\gamma_{\mathrm{p}}^{2}-1\right)^{(s+1) / 2}} \sigma_{\pi^{ \pm}}\left(\gamma_{\mathrm{p}}\right) F_{\mathrm{e}}\left(\gamma_{\mathrm{e}}, \gamma_{\mathrm{p}}\right) \mathrm{d} \gamma_{\mathrm{p}} \\
Q_{\mathrm{g}}\left(E_{\mathrm{g}}, r\right)= & n_{\mathrm{th}, 0} K_{\mathrm{RP}, 0}\left[1+\left(\frac{r}{r_{\mathrm{c}, \mathrm{th}}}\right)^{2}\right]^{-2 q_{\mathrm{th}}} \int_{\gamma_{\min }}^{\infty} c \beta\left(\gamma_{\mathrm{p}}\right) \\
& \times \frac{\gamma_{\mathrm{p}}}{\left(\gamma_{\mathrm{p}}^{2}-1\right)^{(s+1) / 2}} \sigma_{\pi^{0}}\left(\gamma_{\mathrm{p}}\right) F_{\mathrm{g}}\left(E_{\mathrm{g}}, \gamma_{\mathrm{p}}\right) \mathrm{d} \gamma_{\mathrm{p}}
\end{aligned}
$$

where $\sigma_{\pi^{ \pm}}$and $\sigma_{\pi^{0}}$ are the cross sections for the production of charged and neutral pions, respectively, in proton-proton collisions, and the functions $F_{\mathrm{e}}$ and $F_{\mathrm{g}}$ are given by

$$
F_{\mathrm{e}}\left(\gamma_{\mathrm{e}}, \gamma_{\mathrm{p}}\right)=\int_{\gamma_{\pi}^{\min }}^{\gamma_{\pi}^{\max }} \mathrm{d} \gamma_{\pi^{ \pm}} F_{\mathrm{e}}\left(\gamma_{\mathrm{e}}, \gamma_{\pi^{ \pm}}\right) F_{\pi^{ \pm}}\left(\gamma_{\pi^{ \pm}}, \gamma_{\mathrm{p}}\right)
$$

where $F_{\mathrm{e}}\left(\gamma_{\mathrm{e}}, \gamma_{\pi^{ \pm}}\right)$is the electron energy distribution function from pions, and $F_{\pi^{ \pm}}\left(\gamma_{\pi^{ \pm}}, \gamma_{\mathrm{p}}\right)$ is the charged pion energy distribution function;

$$
F_{\mathrm{g}}\left(E_{\mathrm{g}}, \gamma_{\mathrm{p}}\right)=\int_{E_{\pi}^{\min }}^{\gamma_{\pi}^{\max }} \mathrm{d} \gamma_{\pi^{0}} F_{\mathrm{g}}\left(E_{\mathrm{g}}, \gamma_{\pi^{0}}\right) F_{\pi^{0}}\left(\gamma_{\pi^{0}}, \gamma_{\mathrm{p}}\right)
$$

where $F_{\mathrm{g}}\left(E_{\mathrm{g}}, \gamma_{\pi^{0}}\right)$ is the photon energy distribution function from pions and $F_{\pi^{0}}\left(\gamma_{\pi^{0}}, \gamma_{\mathrm{p}}\right)$ is the neutral pion energy distribution function. The distribution functions in (11) and (12) used here are taken from Moskalenko \& Strong (1998).

Given a value of $\xi_{0}$, the $G R$ spectral brightness and luminosity can be obtained immediately from integration of (10) out to a maximum radius $R$. For the $S R E$, in order to obtain their number density as a function of energy and radius, $n_{\mathrm{e}}\left(\gamma_{\mathrm{e}}, r\right)$, it is necessary to solve the equation that locally governs (spatial diffusion can be regarded as irrelevant in this context, see e.g. Sarazin 1999) the balance between injection and energy losses,

$\frac{\partial}{\partial \gamma_{\mathrm{e}}}\left[b\left(\gamma_{\mathrm{e}}, r\right) n_{\mathrm{e}}\left(\gamma_{\mathrm{e}}, r\right)\right]=-Q_{\mathrm{e}}\left(\gamma_{\mathrm{e}}, r\right)$

whose solution is given by

$n_{\mathrm{e}}\left(\gamma_{\mathrm{e}}, r\right)=\frac{1}{b\left(\gamma_{\mathrm{e}}, r\right)} \int_{\gamma_{\mathrm{e}}}^{\infty} Q_{\mathrm{e}}\left(\gamma_{\mathrm{e}}^{\prime}, r\right) \mathrm{d} \gamma_{\mathrm{e}}^{\prime}$.

In (13) and (14), $b\left(\gamma_{\mathrm{e}}\right) \equiv-\mathrm{d} \gamma_{\mathrm{e}} / \mathrm{d} t$ is proportional to the sum of the square of $B_{\mathrm{mwb}}$, the magnetic field corresponding to the energy density of the cosmic microwave background (IC losses) and the square of $B(r)$. The latter quantity is not fixed a priori, but will be derived through an iterative process, when the $S R E$ is requested to reproduce the radio brightness distribution for any given cluster. Then a radial behavior of the magnetic field will univocally emerge, and the normalization $B(r=0) \equiv B_{0}$ will follow from the adopted value of $\xi_{0}$. As a reference value for the maximum in the $G R$ emission to be expected, $\xi_{0}=0.3$ will be adopted as representative of the most extreme conditions described in Sect. 2 with regard to the $R P$ acceleration.

\section{Four clusters and the brightness profile of their radio halos}

The exercise is applied to four clusters as examples. Their names and properties are summarized in Tables 1 and 2. In Table 1, in a sequence, after redshift and temperature, the three parameters $n_{\mathrm{th}, 0}, r_{\mathrm{c}, \mathrm{th}}$, and $q_{\mathrm{th}}$ are given, which quantitatively define the density distribution $n_{\text {th }}$, according to (7). As in Paper I, the radial (azimuthally averaged) brightness distribution at frequency $v$ was fitted with a functional form similar to the one usually adopted for the X-ray brightness, namely (the subscripts $R$ here mean "radio")

$S_{v}(\theta)=S_{v, 0}\left[1+\left(\frac{\theta}{\theta_{\mathrm{c}, \mathrm{R}}}\right)^{2}\right]^{-q_{\mathrm{R}}^{\prime}}$,

where $\theta$ is the angular distance from the center. Under the assumption of spherical symmetry, the profile of the radio emissivity is similar in form to $n_{\mathrm{th}}(7)$, with $q_{\mathrm{R}}=q_{\mathrm{R}}^{\prime}+1 / 2$ 
Table 1. The four clusters, X-ray properties.

\begin{tabular}{cccccc}
\hline \hline & $z$ & $\begin{array}{c}k T \\
(\mathrm{keV})\end{array}$ & $\begin{array}{c}n_{\mathrm{th}, 0} \\
\left(h_{70}^{1 / 2} \mathrm{~cm}^{-3}\right)\end{array}$ & $\begin{array}{c}r_{\mathrm{c}, \mathrm{th}} \\
\left(h_{70}^{-1} \mathrm{kpc}\right)\end{array}$ & $q_{\text {th }}$ \\
\hline Coma & $0.023[1]$ & $8.2[2]$ & $3.4 \times 10^{-3}[2]$ & $300[2]$ & $1.125[2]$ \\
$\mathrm{A} 2319$ & $0.0557[1]$ & $9.12[3]$ & $7.83 \times 10^{-3}[4]$ & $94[5]$ & $0.765[5]$ \\
$\mathrm{A} 2255$ & $0.0806[1]$ & $7.3[6]$ & $1.72 \times 10^{-3}[7]$ & $410[8]$ & $1.11[8]$ \\
$\mathrm{A} 2163$ & $0.203[1]$ & $14.6[9]$ & $7.87 \times 10^{-3}[9]$ & $220[9]$ & $0.93[9]$ \\
\hline
\end{tabular}

References: [1] Struble \& Rood (1999); [2] Briel et al. (1992); [3] Arnaud \& Evrard (1999); [4] Trevese et al. (2000); [5] Feretti et al. (1997b); [6] David et al. (1993); [7] Jones \& Forman (1984); [8] Feretti et al. (1997a); [9] Elbaz et al. (1995).

Table 2. The four clusters, parameters of the radio halo.

\begin{tabular}{ccccccc}
\hline \hline & $\alpha_{\mathrm{R}}$ & $\begin{array}{c}F(1.4 \mathrm{GHz}) \\
(\mathrm{Jy})\end{array}$ & $\begin{array}{c}r_{\mathrm{c}, \mathrm{R}} \\
\left(h_{70}^{-1} \mathrm{kpc}\right)\end{array}$ & $q_{\mathrm{R}}$ & $\begin{array}{c}R_{\mathrm{h}} \\
\left(h_{70}^{-1} \mathrm{kpc}\right)\end{array}$ & $\begin{array}{c}\theta_{\mathrm{h}} \\
(\operatorname{arcmin})\end{array}$ \\
\hline Coma & $1.35[1]$ & $0.64[1]$ & 670 & 4.4 & $900[1]$ & 64 \\
$\mathrm{~A} 2319$ & $1.8:[2]$ & $0.153[2]$ & 37 & 0.98 & $470[2]$ & 16 \\
$\mathrm{~A} 2255$ & $1.7:[3]$ & $0.043[3]$ & 470 & 3.0 & $670[3]$ & 16 \\
$\mathrm{~A} 2163$ & $1.18:[4]$ & $0.155[5]$ & 220 & 1.4 & $1,050[4]$ & 12 \\
\hline
\end{tabular}

References: [1] Deiss et al. (1997); [2] Feretti et al. (1997b); [3] Feretti et al. (1997a); [4] Feretti et al. (2004b); [5] Feretti et al. (2001).

and $r_{\mathrm{c}, \mathrm{R}}=\theta_{\mathrm{c}, \mathrm{R}} D, D$ being the cluster angular distance. These are the two quantities measured at $v=1.4 \mathrm{GHz}$ in Table 2, along with the flux at the same frequency, the radius $R_{\mathrm{h}}$, and the angular size $\theta_{\mathrm{h}}$ of the radio halo. The spectral index $\alpha_{\mathrm{R}}$ in the second column is fairly well-determined only for the Coma cluster; for the three other objects, the value is given with a double dot, to remind the reader that it has not been measured well yet (the flux density data available will appear in Figs. 1-4). Note that the value of $s=2.7$, chosen in Sect. 2 as a reference energy spectral slope of the $R P$, at equilibrium yields a slope of the $S R E$ that almost exactly matches the radio spectral index of Coma (Fig. 1).

\section{Numerical results}

The results are summarized in Table 3 and illustrated in Figs. 1-5. The following quantities are given in Table 3: the number density of the $R P$ at the center, $n_{\mathrm{RP}, 0}$, the value of $B_{0}$ for which the radio flux is best reproduced by the $S R E$ alone, the gamma ray flux from $\pi^{0}$ decay, $f_{\mathrm{g}}(100 \mathrm{MeV})$, obtained integrating out to the radius of the radio-halo $R_{\mathrm{h}}$, the gamma ray luminosity $L_{\mathrm{g}}$ in the band 0.1 to $10 \mathrm{GeV}$, and next to it, for comparison, the radio luminosity approximated as $1.4(\mathrm{GHz}) \times$ $L(1.4 \mathrm{GHz})$. The quantity $\tilde{B}_{0}$ in the fourth column is the reduced mean value of $B$ if "small-scale" scalar fluctuations (see Paper I) are admitted to exist on the order of $\left\langle(\delta B)^{2}\right\rangle / B^{2}=1$ throughout a cluster.

In the upper panels of Figs. 1 to 4, the radial behaviors of $P_{\text {th }}$, $P_{\mathrm{RP}}$, and of the magnetic field pressure are shown out to $R_{\mathrm{h}}$. Note that the magnetic pressure remains lower than the other two everywhere. In the lower panels of the same figures, along with the observational data, the radio spectral flux density is drawn for three values of $B_{0}$, the one given in Table 3 and two more, to illustrate the sensitivity of the normalization to this quantity.

In Fig. 5 the spectral density distributions of the gamma rays from $\pi^{0}$ decay of all four clusters are given. Note that the expected flux scales linearly with $\xi_{0}$, and the values in the figure correspond to $\xi_{0}=0.3$. For comparison the sensitivity curves are shown for the experiment EGRET onboard the satellite Compton-GRO and for the future AGILE and GLAST missions, for an exposure time as reported in the caption. Although
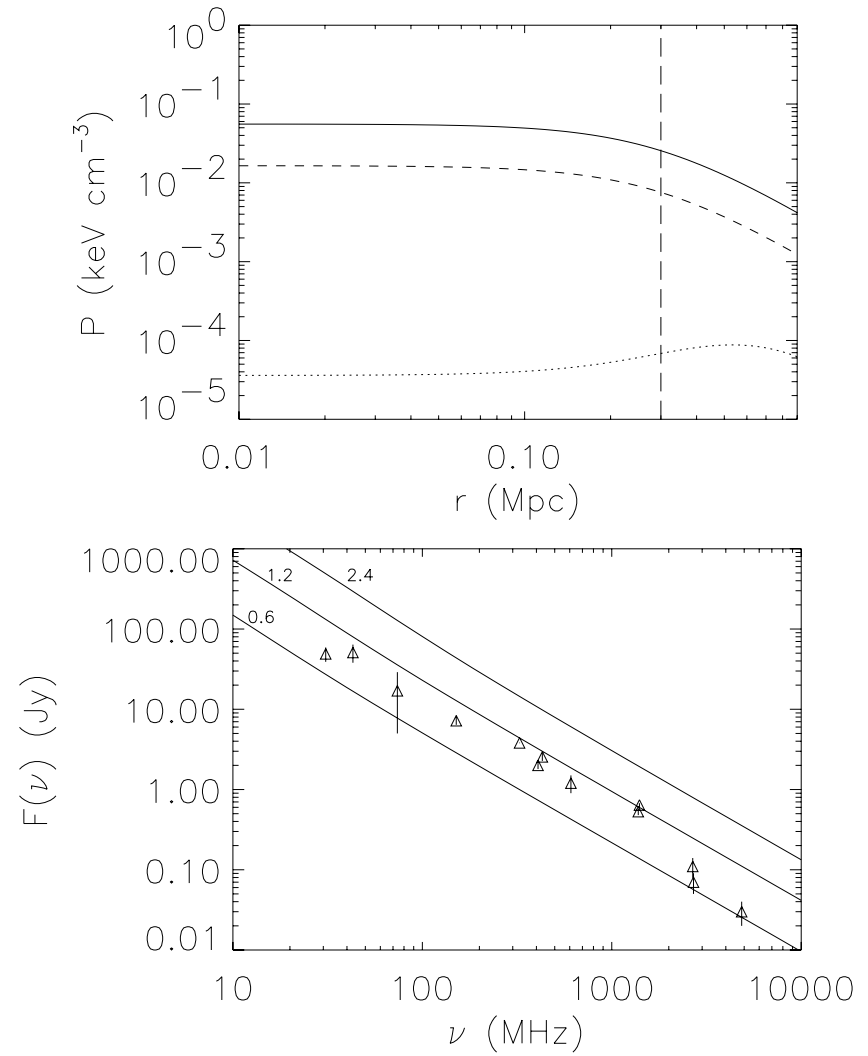

Fig. 1. Coma cluster, $\xi_{0}=0.3, s=2.7$. Upper panel: $P_{\text {th }}$ (full line), $P_{\mathrm{RP}}$ (dashed line), and the magnetic field pressure (dotted line), with $B_{0}=1.2 \mu \mathrm{G}$, as a function of distance from the center. The vertical dashed line marks the position of $r_{\mathrm{c}, \mathrm{th}}$. Lower panel: the SRE radio emission spectra labelled with three values $B_{0}$ (in $\mu \mathrm{G}$ ). The data points are from Thierbach et al. (2003).

the curves apply to point-like sources, note that the angular resolution (on-axis) of GLAST is about 60 arcmin at $1 \mathrm{GeV}$ (from http://www-glast.slac.stanford.edu), hence it should also apply to the extended sources considered here (see Table 2 for the angular size of the radio halos). It is evident that out of the four clusters considered, only the Coma cluster emission could 

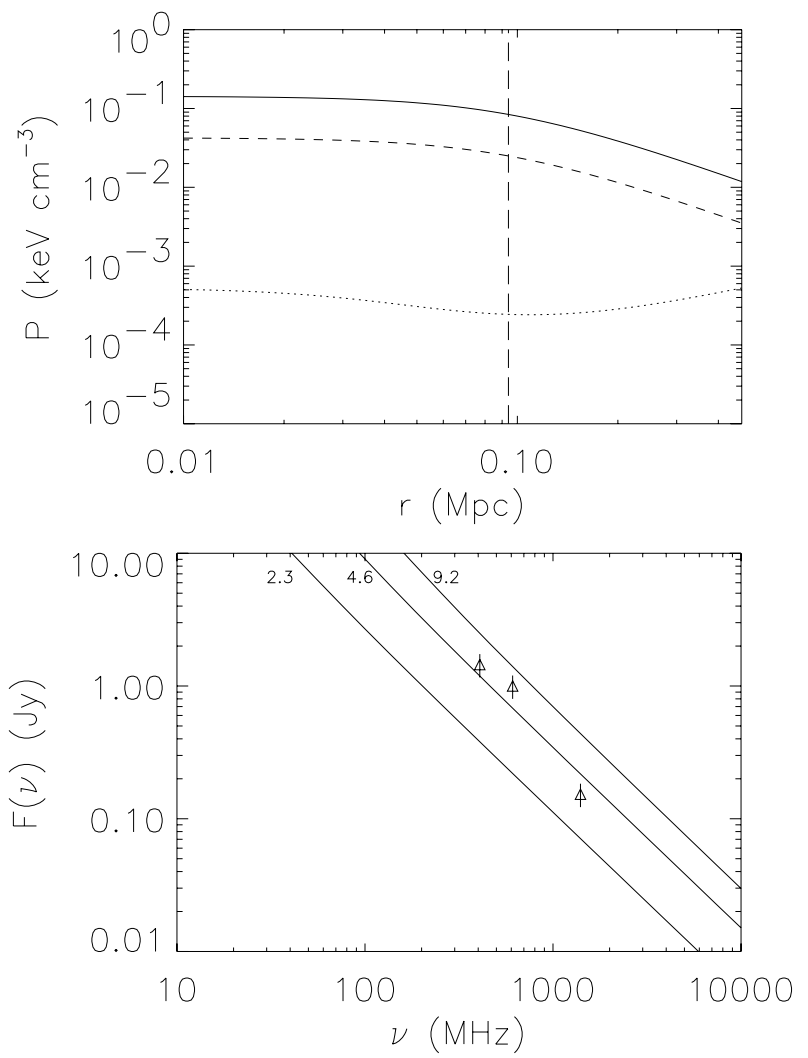

Fig. 2. A2319. Same as Fig. 1, except for: upper panel, $B_{0}=4.6 \mu \mathrm{G}$. Lower panel, data without error bars from Feretti et al. (1997b), with $20 \%$ error bars added arbitrarily.

be significantly detected by GLAST, even if the value of $\xi_{0}$ were lower than 0.3, down to about 0.1 . With $\xi_{0}=0.3$, Coma could be marginally detected by AGILE, while A2319 and A2163 could be marginally detected by GLAST.

The discussion of these results is postponed to Sect. 7, after considering a case of emission, in the ultraviolet, which has been proposed in the literature as due to $S R E$.

\section{SRE and the EUV excess in the Coma cluster}

Within 25 arcmin of the center of the Coma cluster, the EUVE satellite detected an emission in the far UV band 130-180 eV, as reported in Lieu et al. (1996) and Bowyer et al. (1999, 2004). It exceeds the extrapolation of the thermal emission measured in the X-rays. The origin of this excess is controversial (Lieu et al. 1996; Enßlin \& Biermann 1998; Brunetti et al. 2001; Bowyer et al. 2004), here only the hypothesis put forward by Bowyer et al. (2004) (see also Kuo et al. 2005, for a generalization to the clusters as a class) will be considered, namely that, in the first place, the emission mechanism is IC on the microwave background and, in the second, that the electrons have a secondary origin. The reason for inserting this issue into the present paper follows from the fact that the UV measurement, albeit essentially monochromatic and without spectral information, is spatially resolved; hence, under the IC and spherical symmetry assumptions, both the number and the radial distribution of the electrons, whose energy must be $\gamma_{\mathrm{e}} \sim 300$, can be derived in absolute terms. The amount of parent $R P$ can therefore be derived, and their pressure compared with the thermal pressure to verify if the secondary origin of the electrons remains tenable.
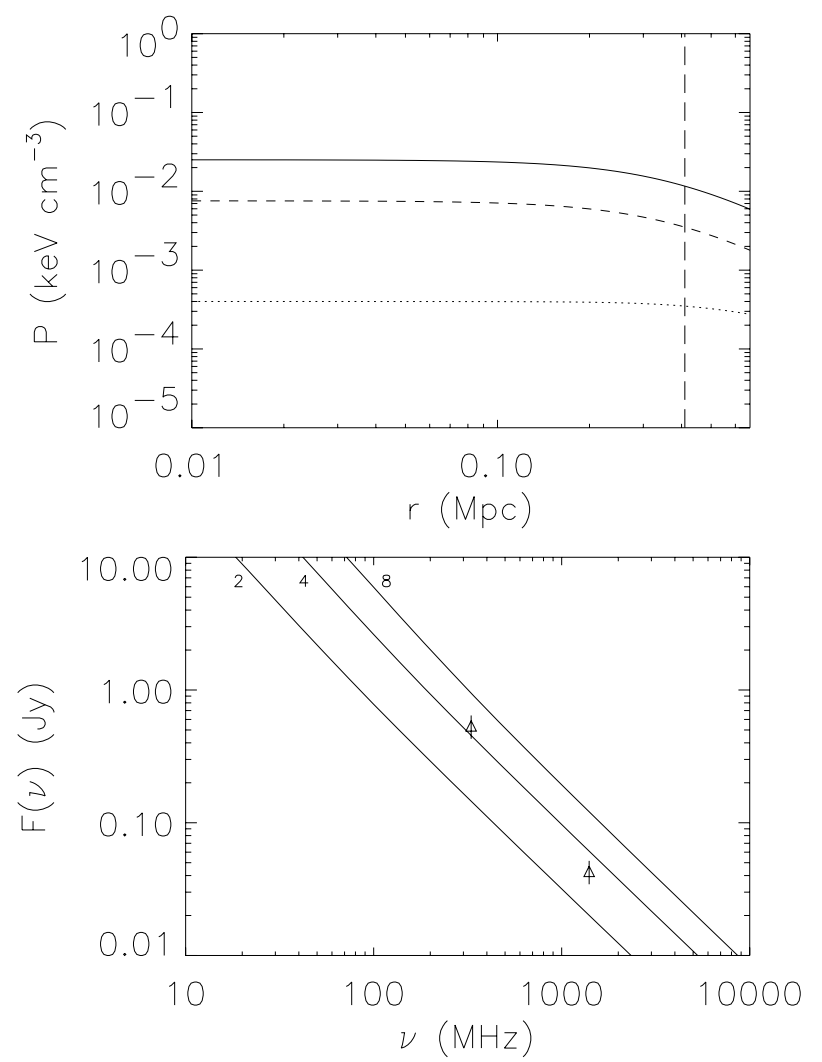

Fig. 3. A2255. Same as Fig. 1, except for: upper panel, $B_{0}=4 \mu \mathrm{G}$. Lower panel, data without error bars from Feretti et al. (1997a), with $20 \%$ error bars added arbitrarily.
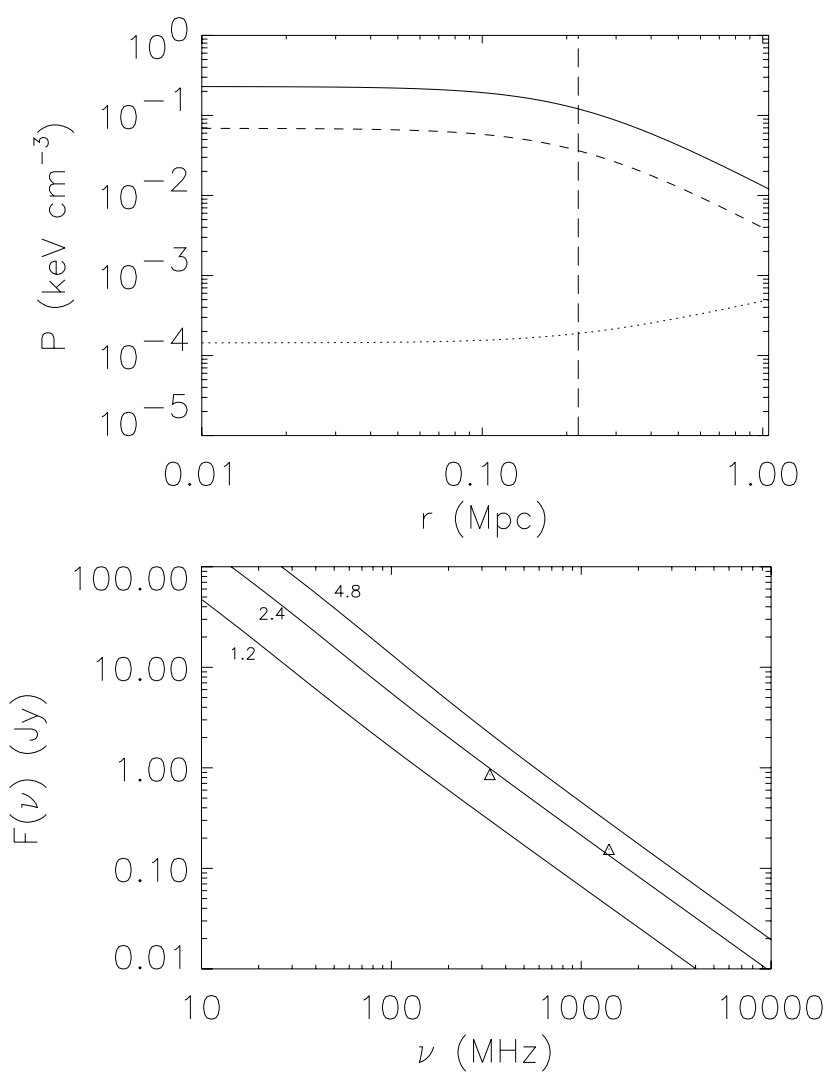

Fig. 4. A2163. Same as Fig. 1, except for: upper panel, $B_{0}=2.4 \mu \mathrm{G}$. Lower panel, data from Feretti et al. (2001, 2004b), whose error bars are within the height of the two triangles. 
Table 3. Numerical results.

\begin{tabular}{ccccccc}
\hline \hline & $\begin{array}{c}n_{\mathrm{RP}, 0} \\
\left(\mathrm{~cm}^{-3}\right)\end{array}$ & $\begin{array}{c}B_{0} \\
(\mu \mathrm{G})\end{array}$ & $\begin{array}{c}\tilde{B}_{0} \\
(\mu \mathrm{G})\end{array}$ & $\begin{array}{c}f_{\mathrm{g}}(100 \mathrm{MeV}) \\
\left(\mathrm{cm}^{-2} \mathrm{~s}^{-1} \mathrm{GeV}^{-1}\right)\end{array}$ & $\begin{array}{c}L_{\mathrm{g}}(0.1-10 \mathrm{GeV}) \\
\left(\mathrm{erg} \mathrm{s}^{-1}\right)\end{array}$ & $\begin{array}{c}v L_{\mathrm{R}}(1.4 \mathrm{GHz}) \\
\left(\mathrm{erg} \mathrm{s}^{-1}\right)\end{array}$ \\
\hline Coma & $1.1 \times 10^{-5}$ & 1.2 & 0.8 & $8.4 \times 10^{-8}$ & $1.3 \times 10^{43}$ & $9.9 \times 10^{39}$ \\
$\mathrm{~A} 2319$ & $2.4 \times 10^{-5}$ & 4.6 & 3.2 & $8.6 \times 10^{-9}$ & $8.6 \times 10^{42}$ & $1.5 \times 10^{40}$ \\
$\mathrm{~A} 2255$ & $6.0 \times 10^{-6}$ & 4.0 & 2.8 & $2.2 \times 10^{-9}$ & $5.4 \times 10^{42}$ & $9.7 \times 10^{39}$ \\
$\mathrm{~A} 2163$ & $2.0 \times 10^{-5}$ & 2.4 & 1.7 & $6.0 \times 10^{-9}$ & $1.0 \times 10^{44}$ & $2.6 \times 10^{41}$ \\
\hline
\end{tabular}

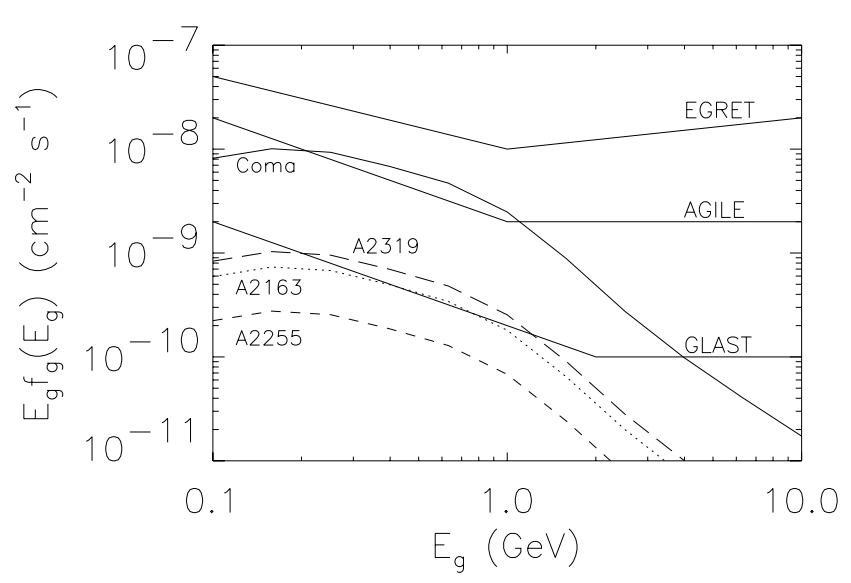

Fig. 5. Spectral energy distribution of the gamma ray flux from $\pi^{0}$ decay expected in the case illustrated for each cluster in Figs. 1 to 4 . The sensitivity of EGRET, AGILE, and GLAST is drawn for comparison purposes and corresponds to a $5 \sigma$ detection in a full-year sky survey (from http://people.roma2.infn.it/ agile/).

The profile of the UV surface brightness $S_{\mathrm{UV}}(\theta)$ can be described with the functional form of Eq. (15), and it turns out (as pointed out by Bowyer et al. 2004) to be very similar to that of the thermal X-ray emission. A best fit with $\theta_{\mathrm{c}, \mathrm{UV}}=\theta_{\mathrm{c}, \mathrm{X}}=$ 10.5 arcmin yields an exponent $q_{\mathrm{UV}}^{\prime}=1.92 \pm 0.20\left(\chi_{\text {red }}^{2}=0.64\right.$ with 11 d.o.f.), consistent within $1 \sigma$ with $q_{\mathrm{X}}^{\prime}=1.75$ (Briel et al. 1992). The emissivity is given (see Sect. 4) by a functional form like (7), with $r_{\mathrm{c}, \mathrm{UV}}=r_{\mathrm{c} \text {,th }}$ and $q_{\mathrm{UV}}=q_{\mathrm{UV}}^{\prime}+1 / 2=2.42 \pm 0.20$ : in the following $q_{\mathrm{UV}}=2.42$ will be used. Since the emissivity is proportional to the number density of the electrons $n_{\mathrm{e}}$ with $\gamma_{\mathrm{e}}$ about 300 , this quantity will have the same radial dependence:

$n_{\mathrm{e}}(r)=n_{\mathrm{e}}(0)\left[1+\left(\frac{r}{r_{\mathrm{c}, \mathrm{th}}}\right)^{2}\right]^{-2.42}$.

The $R E$ with $\gamma_{\mathrm{e}}$ about 300 would emit synchrotron radiation at $v \sim 0.4(B / \mu \mathrm{G}) \mathrm{MHz}$, an inaccessible window. As pointed out already by Bowyer $\&$ Berghöfer (1998), the radial behavior (16) is likely to be very dissimilar from that of the $R E$ responsible for the observed radio emission; otherwise, it would imply a magnetic field increasing with $r$ out to at least $2 / 3 R_{\mathrm{h}}$ for the radio brightness distribution of Coma C.

Given the $S R E$ density distribution (16), one can resort to the population of the parent $R P$ by inverting the procedure described in Sect. 4. The first step consists in obtaining $Q_{\mathrm{e}}\left(\gamma_{\mathrm{e}}, r\right)$ from Eq. (13). Concerning the energy-loss rate, $b\left(\gamma_{\mathrm{e}}, r\right)$, it now consists of two terms, the Coulomb loss rate $b_{\mathrm{c}}$, which depends on $r$ since $b_{\mathrm{c}} \propto n_{\mathrm{th}}$, and the IC loss rate $b_{\mathrm{IC}}$, which is constant in space and corresponds to the loss rate in an equivalent magnetic field $B_{\mathrm{mwb}}=3(1+z)^{2} \mu \mathrm{G}$. The synchrotron losses depend on the unknown value and radial behavior of the magnetic field: ignoring these losses does not affect the conclusion, as will be clear later

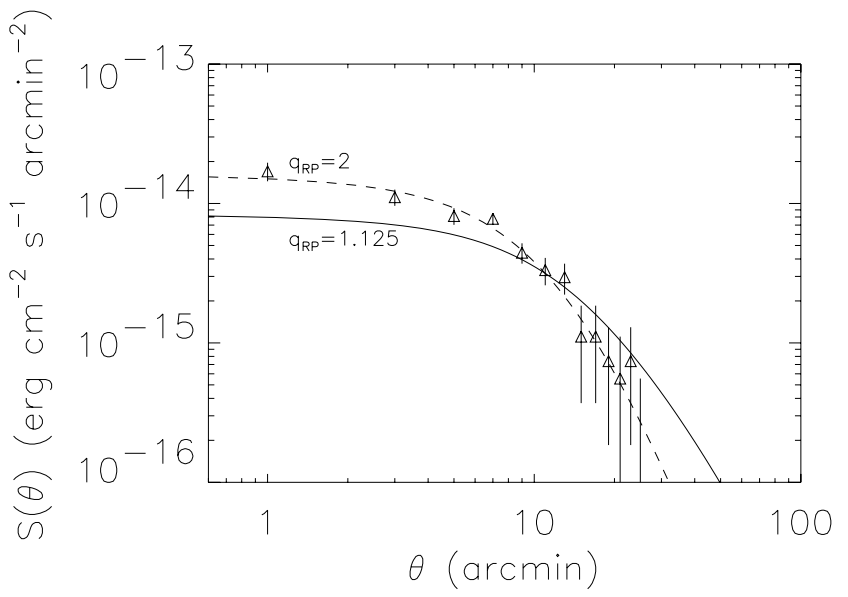

Fig. 6. The UV brightness profile from $S R E$ for two values of $q_{\mathrm{RP}}$ (see text). The data are from Bowyer et al. (2004).

on. Using the expression for $b_{\mathrm{c}}$ given in Sarazin (1999), it turns out that (for $n_{\text {th }}$ constant with time) at $r$ much less than $r_{\mathrm{c}}$ and for electrons with $\gamma_{\mathrm{e}} \sim 300, b_{\mathrm{c}}$ was equal to $b_{\mathrm{IC}}$ at $z^{*}=0.384$, and their lifetime was $2.1 \mathrm{Gyr}$ at that epoch. The time elapsed since then up to the epoch corresponding to the redshift of the Coma Cluster - for $H_{0}=70 \mathrm{~km} \mathrm{~s}^{-1} \mathrm{Mpc}^{-1}, \Omega_{\mathrm{m}}=0.3$, and $\Omega_{\Lambda}=0.7-$ is $3.4 \mathrm{Gyr}$, therefore the UV emission observed now from the innermost region of the cluster is due to electrons affected more by Coulomb than by IC losses. At larger distances from the center the latter dominate instead.

Equation (13) contains the spectral energy distribution of the electrons, which is unknown. The calculations were carried out in full, that is by using Eq. (13), in combination with Eq. (9), adopting two different values of $s$ for the $R P$, namely 2.7 and 2.3.

The radial distribution of the parent $R P$, which reproduces the UV brightness profile fairly well (see Fig. 6), is given by

$n_{\mathrm{RP}}(r) \propto\left[1+\left(\frac{r}{r_{\mathrm{c}}}\right)^{2}\right]^{-q_{\mathrm{RP}}}$,

with $r_{\mathrm{c}}=r_{\mathrm{c} \text {,th }}$ and $q_{\mathrm{RP}}=2$ (irrespective of which one of the two values of $s$ is used). The exponent $q_{\mathrm{RP}}$ is rather well constrained: as shown in Fig. 6, the UV brightness distribution could not be reproduced if the $R P$ shared the same radial distribution with the thermal gas, namely if $q_{\mathrm{RP}}=q_{\mathrm{th}}=1.125$. This would, notably, turn out to be the case if the Coulomb losses had been ignored and only the spatially "constant" IC losses taken into account. The result obtained implies that the quantity $\xi(r)$, defined by (5) is not constant and that it is maximum at the center.

The normalization of Eq. (17) and the corresponding $P_{\mathrm{RP}}$ depends on the exponent $s$. The values of $\xi(r=0)$ for the two choices of $s$, obtained by integrating the $R P$ spectrum down to the $\gamma_{\min }$ given by Eq. (2), are given in Table 4 and are much higher than unity for both values of $q_{\mathrm{RP}}$ in Fig. 6. The inevitable 
Table 4. The value of $\xi(r=0)$, for two choices of the exponents $s$ and $q_{\mathrm{RP}}$, needed to explain the UV excess in the Coma Cluster as due to $S R E$.

\begin{tabular}{|c|c|c|c|}
\hline$s=2.7$ & $\xi(r=0)$ & $s=2.3$ & $\xi(r=0)$ \\
\hline$q_{\mathrm{RP}}=1.125$ & 9 & $q_{\mathrm{RP}}=1.125$ & 7 \\
\hline$q_{\mathrm{RP}}=2$ & 21 & $q_{\mathrm{RP}}=2$ & 15 \\
\hline
\end{tabular}

conclusion, which would be even stronger if the synchrotron losses were non-negligible, is that the hypothesis of a secondary origin of the electrons is not tenable in this case.

Surprisingly, Bowyer et al. (2004) reach the opposite conclusion. This follows, partly, from their decision to ignore the Coulomb losses, in addition to the synchrotron losses. But this implies at most a difference in the value of $\xi$ by a factor five. Since other approximations only have marginal effects, one is led to conclude that their result is affected by a material error. In this respect, it is worth mentioning that Miniati et al. (2001b) conclude that the UV excess due to SRE should be lower than the measured value, on the basis of a ratio $1 / 2$ between the energy content in $C R$ and in thermal gas obtained with numerical simulations of the acceleration processes. All the more so, when account is taken of the fact that the value adopted for comparison is the one reported by Bowyer et al. (1999), which is a factor 2.7 lower than the one later presented in Bowyer et al. (2004).

\section{Discussion}

It is convenient to start by focusing the discussion on the central value $B_{0}$ and the radial behavior of the magnetic field. The values of $B_{0}$ in Table 3 span an interval from 1.2 (Coma) to $4.6 \mu \mathrm{G}$ (A2319). In all four cases, the corresponding pressure is well below the $C R$ pressure. If the "true" magnetic field strength were substantially smaller or larger, the consequence for the SRE in the central region would be, in the first case, that their contribution must be negligible (otherwise $\xi_{0}$ should be unacceptably large), in the second case that their contribution is even more likely to be important, since $\xi_{0}$ could take a value lower than 0.3 , which is the reference value adopted above to approximately represente the "maximum" $R P$ content. A comparison with the current estimates of $B_{0}$ from FR measurements is therefore in order.

None of the values of $B_{0}$ in Table 3 seems to be inconsistent with typical FR estimates in the inner regions of clusters, but one must take into account (see Paper I and references therein for a brief presentation of this issue) the rather large uncertainties affecting the FR results, which are mainly related to the approximations adopted for the spectrum of the field fluctuations as a function of their scale length. In particular, the value for the Coma cluster is in fair agreement with the estimate obtained by Kim et al. (1990) using background sources, but not with the one obtained by Feretti et al. (1995), using one extended source within the cluster, which could be as large as $\sim 8 \mu \mathrm{G}$, if a very small scale length for field reversals is adopted, of the order of $1 \mathrm{kpc}$. The value recently derived for A2255 (Govoni et al. 2006), using three extended sources within the cluster and a more sophisticated approach than in Feretti et al. (1995), is $2.5 \mu \mathrm{G}$ (the confidence level is not explicitely given in the paper), which is not far from the $4.0 \mu \mathrm{G}$, and practically equal to $\tilde{B}_{0}$, in Table 3 . For the two other clusters the "individual" FR measurements available are not reliable. It must, in addition, be noticed that, in the frequency range around $1.4 \mathrm{GHz}$, the $S R E$ spectral energy slope is about 3.7 (for the choice made of $s=2.7$ ), because of the radiative losses, and the radio emissivity scales as $B^{(3.7+1) / 2}=B^{2.35}$ : thus, it is very sensitive to the $B$ value, as also illustrated in Figs. 1 to 4 . Therefore, so long as a very robust FR estimate for each individual cluster is lacking, a generic agreement is by no means a strong enough argument for concluding that the $S R E$ might always be an important, if not the dominant, contribution. Moreover, a very relevant uncertainty on the values of $B$ for the three clusters other than Coma stems from the large uncertainty on the radio spectral slope, hence on their specific value of $s$ and consequently on the $R P$ pressure for a fixed value of $\xi_{0}$ (Eqs. (3)-(5)). At present, it can only be stated that the above-mentioned conclusion cannot be excluded for the inner region of a cluster and its radio halo.

The situation is plainly different at distances from the center that are much greater than $r_{\mathrm{c}, \text { th }}$. In the three objects where $R_{\mathrm{h}}$ is several times larger than the core radius, Figs. 1, 2, and 4 show that the magnetic pressure, although it remains well below the $C R$ pressure, tends to increase, a behavior that is physically embarrassing (see also Brunetti 2003 and Pfrommer \& Enßlin 2004 for the case of Coma). Rather than sharply concluding that the $S R E$ should therefore be regarded as a minor component of the bulk $R E$, it is perhaps more sensible to consider the possibility that the contribution of the PRE might become dominant at large radii, thus providing a way to get around this problem. To qualify this possibility, one can, for instance, assume a continuous injection of fresh $P R E$ throughout the cluster, described by a source function $Q_{\mathrm{ep}}\left(\gamma_{\mathrm{e}}, r\right)$, with a radial dependence of the form (8) adopted above for the accumulated $R P$ and an energy dependence with the same index $s$. The solution (14) would then give the equilibrium distribution, whose energy dependence is practically the same as that of the SRE. One of the results for the case of Coma is illustrated in Fig. 7. Here the PRE and SRE contributions at the center are assumed to have equal weight (that is, the quantity $\xi_{0}$ was reduced to 0.15 to ensure that the SRE central density amounts to half the value derived previously, while the value of $B_{0}$ obviously remains the same, $1.2 \mu \mathrm{G}$ ). The rise in magnetic pressure present in Fig. 1 has gone away almost completely, except for the mild bump around $400 \mathrm{kpc}$, which is evidently the artificial consequence of $r_{\mathrm{c}, \mathrm{R}}$ being about twice $r_{\mathrm{c}, \text { th }}$ (see Tables 1 and 2), together with the prescription $r_{\mathrm{c}, \mathrm{RP}}=r_{\mathrm{c} \text {,th }}$ adopted in Sect. 3. In conclusion, to obtain an acceptable radial behavior of the magnetic field, one must resort to $P R E$ at large distances from the center, but they need not necessarily be the dominant fraction within $r_{\mathrm{c}, \text { th }}$, where the production of $S R E$ is most effective.

Observationally, the case of the UV excess analyzed in Sect. 6 is very instructive. If the surface brightness of the IC emission by the electrons responsible for the radio emission could be measured in the hard X-ray band, the same procedure could be followed to verify whether or not the amount of parent $R P$ is compatible with the condition $\xi$ less than unity everywhere throughout the cluster. A positive answer would not, however, be conclusive as to the secondary origin of the $R E$. This issue can only be addressed directly through measurements of the collisionally-produced GR. This brings one back to the key issue of the effective $C R$ content of clusters.

In this respect, a final important remark is due on the apparent dichotomy between those clusters endowed with a radio halo and those that are not. In the current literature (Feretti et al. 2004a), a correlation is emphasized between the existence of a radio halo and the evidence of ongoing merging phenomena. This correlation speaks in favor of a dominant population of $P R E$ everywhere in these clusters, which are being accelerated since 
Table 5. The four clusters studied in this paper (upper part) and a sample of six clusters whitout a radio halo (lower part).

\begin{tabular}{ccccc}
\hline \hline Cluster & $\begin{array}{c}r_{\mathrm{c}, \mathrm{th}} \\
(\operatorname{arcmin})\end{array}$ & $\begin{array}{c}F_{1.4}\left(r \leq r_{\mathrm{c}, \mathrm{h}}\right) \\
(\mathrm{mJy})\end{array}$ & $\begin{array}{c}F_{1.4}\left(r \leq r_{\mathrm{c}, \mathrm{th}}\right) \\
\text { (upper limit, mJy) }\end{array}$ & $\begin{array}{c}F_{\mathrm{g}}\left(0.1-10 \mathrm{GeV}, r \leq r_{\mathrm{c}, \mathrm{th}}\right) \\
\left(\mathrm{cm}^{-2} \mathrm{~s}^{-1}\right)\end{array}$ \\
\hline Coma & 10.5 & 160 & & $6.8 \times 10^{-9}$ \\
$\mathrm{~A} 2319$ & 1.6 & 18 & & $2.7 \times 10^{-10}$ \\
$\mathrm{~A} 2255$ & 4.8 & 25 & & $2.7 \times 10^{-10}$ \\
$\mathrm{~A} 2163$ & 1.2 & 18 & & $2.9 \times 10^{-10}$ \\
\hline A119 & 5.3 & 30 & $\leq 47$ & $1.8 \times 10^{-10}$ \\
$\mathrm{~A} 2063$ & 1.3 & 6 & & $3.8 \times 10^{-11}$ \\
$\mathrm{~A} 1775$ & 1.8 & 6 & & $4.1 \times 10^{-11}$ \\
$\mathrm{~A} 1413$ & 0.7 & 8 & & $5.8 \times 10^{-11}$ \\
A399 & 1.9 & 22 & $\leq 1.6$ & $1.5 \times 10^{-10}$ \\
$\mathrm{~A} 3667$ & 7.9 & 54 & $\leq 209$ & $3.3 \times 10^{-10}$ \\
\hline
\end{tabular}

The X-ray parameters adopted for the predictions in the lower part come from: Cirimele et al. (1997) for A119, A2063, A1775 and A1413; Sakelliou \& Ponman (2004) for A399; Vikhlinin et al. (2001) for A3667. The flux upper limits in A119, A399 derive from Giovannini et al. (2006), the one in A3667 from Feretti (2004).

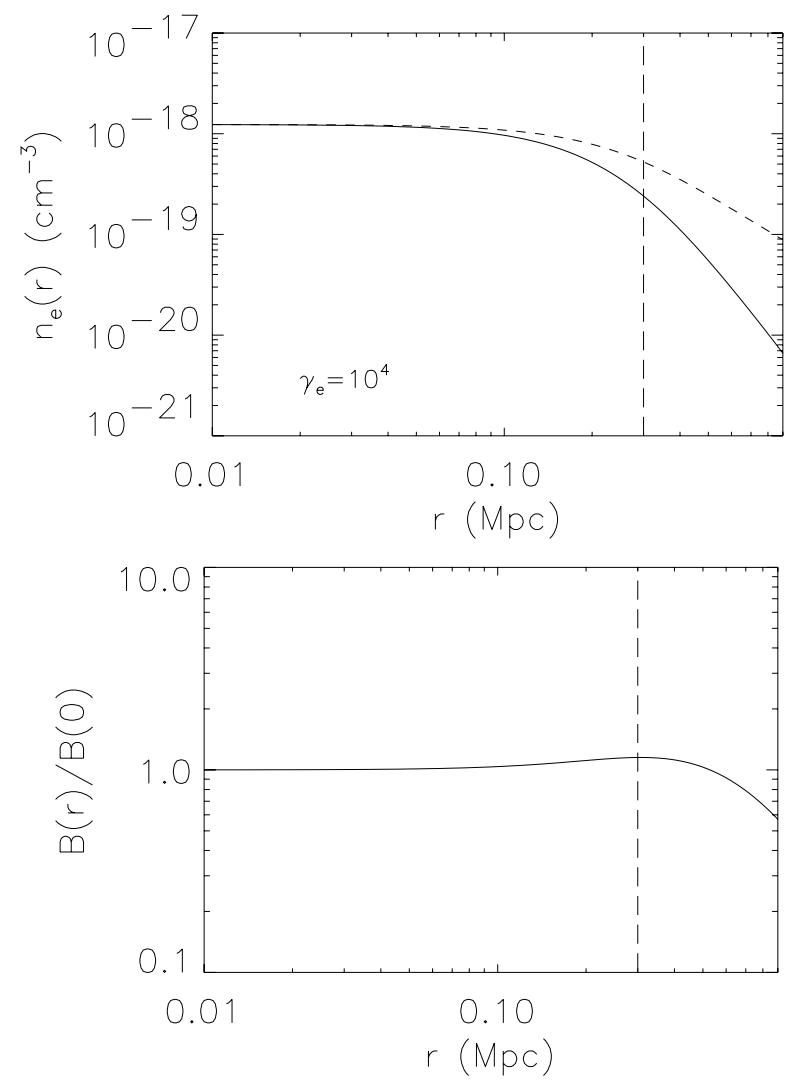

Fig. 7. Upper panel: hypothetical mix of $S R E$, in the case $\xi_{0}=0.15$, with $P R E$ of equal density at the center of the Coma Cluster. The density refers to electrons with $\gamma_{\mathrm{e}}=10^{4}$, the energy corresponding approximately to the synchrotron frequency $v_{\mathrm{c}}=1.4 \mathrm{GHz}$ when $B_{0}=1.2 \mu \mathrm{G}$. Lower panel: the radial behavior of the magnetic field required to reproduce the radio surface brightness. See text for details.

at most one billion years. However, it must be stressed that an attempt to estimate the strength of the magnetic field in those clusters devoid of a diffuse radio emission by combining FR measurements in an ensemble of them (Clarke et al. 2001) resulted in a value of $B$ in the interval 1 to $10 \mu \mathrm{G}$, the same interval of the clusters with a radio halo. The results obtained in Sect. 5 would then suggest that the amount of RP, which have accumulated in both types of clusters all along their ten billion years history, should be significantly lower than the amount corresponding to the reference value $\xi_{0}=0.3$. It is the opinion of the authors of the present paper that more sensitive measurements of a diffuse radio emission in clusters, where it has gone undetected so far (along with more accurate "individual" estimates of $B$ using the FR), might provide very stringent constraints on their $C R$ content. In consideration of the hard fact that the detection of $G R$ from more than just one cluster will be a difficult task even for the mission GLAST, further efforts should be made in the radio window. In order to stimulate such efforts, some clusters, where a diffuse radio emission has gone undetected so far, and which look like promising targets, have been selected (Table 5, lower part). For reasons of homogeneity with the ones discussed in this paper, none of these clusters shows the soft excess usually named "cooling flow". The diffuse radio emission from these clusters, which should be due exclusively to $S R E$, should be typically more concentrated than in the "radio halo" clusters, because of the dependence of the production rate from the product $n_{\text {th }} \times n_{\mathrm{RP}}$. Thus, the $1.4 \mathrm{GHz}$ flux (Table 5, Col. 3) was calculated (according to the prescriptions in Sect. 3) for each of them and on the basis of its X-ray properties, from within $r=r_{\mathrm{c}, \text { th }}$ (Table 5, Col. 2), assuming the reference value 0.3 for $\xi_{0}$, and a value equal to $5 \mu \mathrm{G}$ for $B_{0}$. Note that the predicted flux values scale linearly with $\xi_{0}$ and, in first approximation, with the square of $B_{0}$. In the same table, for comparison, the flux is reported from the corresponding region of the four clusters studied above. Despite the large uncertainties associated with the actual value of $B_{0}$, it is encouraging that the predictions are comparable, in order of magnitude, with the measured values of the four clusters. Furthermore, as reported in the same table (Col. 4), interesting upper limits already exist for three of them. One case seems particularly significant, namely A119, where the prediction and the upper limits are comparable, while some FR measurements are available (Clarke et al. 2001; Feretti et al. 1999) that indicate a $B_{0}$ value of about $5 \mu \mathrm{G}$ in this cluster (Murgia et al. 2004). Thus an improvement by a factor of a few in the radio upper limit could already place a significant constraint on $\xi_{0}$. Also the case of A399, where the upper limit is about ten times lower than the prediction, could be very significant, but the authors are not aware of reliable FR measurements for this object. The last column of Table 5 reports the predicted gamma-ray fluxes in the $0.1-10 \mathrm{GeV}$ interval from within $r=r_{\mathrm{c}, \mathrm{th}}$ : from a comparison with the similarly defined fluxes of the four clusters in the upper part of the table, they appear to be hardly detectable, with the possible exception of A3667. 


\section{Conclusions}

The issue of the CR content in galaxy clusters, which are endowed with a diffuse radio emission called radio halo, was discussed here in terms of the secondary products of collisions with the thermal gas, namely $S R E$ and $G R$. This production is maximized when the density profile of the $R P$ is identical in form to that of the thermal gas. The production rate can be normalized by adopting a value of the ratio between $R P$ and thermal pressure, $\xi_{0}$, constant throughout the cluster out to the radius $R_{\mathrm{h}}$ of the radio halo. This quantity represents the efficiency and output of current models of $C R$ acceleration associated to merging processes in clusters. The value adopted here as a reference is $\xi_{0}=0.3$ to represent the case of maximum efficiency and cumulation over the cluster lifetime. A representative value $s=2.7$ has been chosen for the energy spectral index of the $R P$.

The equilibrium spectrum of the $S R E$ is then constrained to reproduce the observed radio emission, and both the central value $B_{0}$ and the radial behavior of the magnetic field is then uniquely derived. In the four clusters treated, the values of $B_{0}$ fall between 1.2 and $4.6 \mu \mathrm{G}$ (reduced to 0.8 to $3.2 \mu \mathrm{G}$ if scalar fluctuations on the order of $\left\langle(\delta B)^{2}\right\rangle / B^{2}=1$ are admitted): these values are consistent with estimates of the same quantity from FR measurements within clusters in general, but more detailed FR measurements are needed for each cluster individually before a sound conclusion might be drawn. Particularly in view of the high sensitivity of the synchrotron emissivity to the value of $B$.

The radial behavior of $B$, instead, seems unnatural, in that it tends to increase with the radial distance from the center. Rather than definitely exclude a relevant contribution of the $S R E$ to the radio emission also in the central parts of a cluster, this result suggests that $P R E$, accelerated "recently", i.e. within their maximum radiative lifetime, must dominate at large radii. Figure 7 shows, in the case of the Coma cluster, how the mix of the two contributions alleviates the problem of the radial behavior of $B$, even if the contribution of the $S R E$ within the thermal core radius is assumed to be as large as that of the PRE.

The GR production rate in the same conditions described above immediately yields the expected luminosity and flux in the 0.1 to $10 \mathrm{GeV}$. A comparison with the sensitivity curves of the future missions AGILE and GLAST shows that, out of the four clusters considered here, only the Coma cluster could be significantly detected by GLAST, even if $\xi_{0}$ were as small as 0.1 . With $\xi_{0}=0.3$, Coma could be marginally detected by AGILE, while A2319 and A2163 could be marginally detected by GLAST.

The proposal by Bowyer et al. (2004) that the spatiallyresolved UV excess emission in the Coma Cluster, supposedly due to IC scattering by $R E$ on the cosmic microwave background, might originate from $S R E$, is discussed as an instructive example of what could be done if spatially-resolved measurements of the IC emission from the radioemitting electrons, expected in the hard X-rays, were available. The $S R E$ hypothesis is rejected on the grounds of an estimate of $\xi$ that, at the center of the cluster, should be at least one order of magnitude greater than unity.

Finally, the dichotomy between clusters with and without radio halos is presented as an opportunity to test the $C R$ content in clusters in general. This follows from the fact that clusters without a diffuse radio emission, according to FR measurements (Clarke et al. 2001), share a magnetic field of similar strength with those endowed with a radio halo. The results show that, if $\xi_{0}$ were close to the reference value adopted in this paper, the SRE would give rise to a measurable radio emission. Therefore, any effort to improve the limits on diffuse radio emission from clusters in general, and the estimate of their magnetic field through FR measurements, will help to constrain their $C R$ content before the hard task of the future gamma ray experiments can be accomplished.

Acknowledgements. The authors thank G. Giovannini for early discussions of the subject and the referee for constructive comments. S.C. is supported by PRIN-MIUR under contract No.2004027755_003.

\section{References}

Arnaud, M., \& Evrard, A. E. 1999, MNRAS, 305, 631

Blandford, R., \& Eichler, D. 1987, Phys. Rep., 154, 1

Bowyer, S., \& Berghöfer, T. W. 1998, ApJ, 506, 502

Bowyer, S., Berghöfer, T. W., \& Korpela, E. J. 1999, ApJ, 526, 592

Bowyer, S., Korpela, E. J., Lampton, M., \& Jones, T. W. 2004, ApJ, 605, 168

Briel, U. G., Henry, J. P., \& Böhringer, H. 1992, A\&A, 259, L31

Brunetti, G. 2003, in Matter and Energy in Clusters of Galaxies, ed. S. Bowyer, \& C.-Y. Hwang, ASP Conf. Ser., 301, 349

Brunetti, G., Setti, G., Feretti, L., \& Giovannini, G. 2001, New Astr., 6, 1

Burbidge, G. R. 1956, ApJ, 124, 416

Cirimele, G., Nesci, R., \& Trevese, D. 1997, ApJ, 475, 11

Clarke, T. E., Kronberg, P. P., \& Böhringer, H. 2001, ApJ, 547, L111

Colafrancesco, S., Marchegiani, P., \& Perola, G. C. 2005, A\&A, 443, 1 (Paper I)

Colafrancesco, S., Profumo, S., \& Ullio, P. 2006, A\&A, 455, 21

David, L. P., Slyz, A., Jones, C., et al. 1993, ApJ, 412, 479

Deiss, B. M., Reich, W., Lesch, H., \& Wielebinski, R. 1997, A\&A, 321, 55

Dennison, B. 1980, ApJ, 239, L93

Elbaz, D., Arnaud, M., \& Böhringer, H. 1995, A\&A, 293, 337

Enßlin, T. A., \& Biermann, P. L. 1998, A\&A, 330, 90

Feretti, L. 2004 [arXiv: astro-ph/0406090]

Feretti, L., Dallacasa, D., Giovannini, G., \& Tagliani, A. 1995, A\&A, 302, 680

Feretti, L., Böhringer, H., Giovannini, G., \& Neumann, D. 1997a, A\&A, 317, 432

Feretti, L., Giovannini, G., \& Böhringer, H. 1997b, New Astr., 2, 501

Feretti, L., Dallacasa, D., Govoni, F., et al. 1999, A\&A, 344, 472

Feretti, L., Fusco-Femiano, R., Giovannini, G., \& Govoni, F. 2001, A\&A, 373, 106

Feretti, L., Burigana, C., \& Enßlin, T. A. 2004a, New Astr., 48, 1137

Feretti, L., Orrù, E., Brunetti, G., et al. 2004b, A\&A, 423, 111

Fusco-Femiano, R., Orlandini, M., Brunetti, G., et al. 2004, ApJ, 602, L73

Gieseler, U. D. J., Jones, T. W., \& Kang, H. 2000, A\&A, 364, 911

Giovannini, G., Feretti, L., Govoni, F., Murgia, M., \& Pizzo, R. 2006, AN, 327, 563

Govoni, F., \& Feretti, L. 2004, Inter. J. Mod. Phys. D, 13, 1549

Govoni, F., Murgia, M., Feretti, L., et al. 2006, A\&A, 460, 425

Jaffe, W. J. 1977, ApJ, 212, 1

Jones, C., \& Forman, W. 1984, ApJ, 276, 38

Kim, K. T., Kronberg, P. P., Dewdney, P. E., \& Landecker, T. L. 1990, ApJ, 355, 29

Kuo, P.-H., Bowyer, S., \& Hwang, C.-Y. 2005, ApJ, 618, 675

Lieu, R., Mittaz, J. P. D., Bowyer, S., et al. 1996, Science, 274, 1335

Miniati, F., Ryu, D., Kang, H., \& Jones, T. W. 2001a, ApJ, 559, 59

Miniati, F., Jones, T. W., Kang, H., \& Ryu, D. 2001b, ApJ, 562, 233

Moskalenko, I. V., \& Strong, A. W. 1998, ApJ, 493, 694

Murgia, M., Govoni, F., Feretti, L., et al. 2004, A\&A, 424, 429

Perola, G. C., \& Reinhardt, M. 1972, A\&A, 17, 432

Pfrommer, C., \& Enßlin, T. A. 2004, A\&A, 413, 17

Pfrommer, C., Springel, V., Enßlin, T. A., \& Jubelgas, M. 2006, MNRAS, 367, 113

Pieri, L., \& Branchini, E. 2004, Phys. Rev. D, 69, 043512

Rossetti, M., \& Molendi, S. 2004, A\&A, 414, L41

Ryu, D., Kang, H., Hallman, E., \& Jones, T. W. 2003, ApJ, 593, 599

Sakelliou, I., \& Ponman, T. J. 2004, MNRAS, 351, 1439

Sarazin, C. L. 1999, ApJ, 520, 529

Struble, M. F., \& Rood, H. J. 1999, ApJS, 125, 35

Thierbach, M., Klein, U., \& Wielebinski, R. 2003, A\&A, 397, 53

Trevese, D., Cirimele, G., \& De Simone, M. 2000, ApJ, 530, 680

Vikhlinin, A., Markevitch, M., \& Murray, S. S. 2001, ApJ, 549, L47

Willson, M. A. G. 1970, MNRAS, 151, 1 